\title{
Complicated colorings
}

\author{
Assaf Rinot
}

If $\lambda, \kappa$ are regular cardinals, $\lambda>\kappa^{+}$, and $E_{\geq \kappa}^{\lambda}$ admits a nonreflecting stationary set, then $\operatorname{Pr}_{1}(\lambda, \lambda, \lambda, \kappa)$ holds.

\section{Introduction}

The theory of strong colorings was born with Sierpiński's 1933 construction of a symmetric function $c: \mathbb{R}^{2} \backslash \Delta \rightarrow 2$ that does not admit an uncountable monochromatic square. For the construction, one fixes a well-ordering $<_{W}$ of the reals, and contrast it with the usual ordering $<$, by letting $c(x, y)=1$ iff $\left(x<y \& x<_{W} y\right) \vee\left(y<x \& y<_{W} x\right)$. The separability of $\mathbb{R}$ then implies that for every uncountable set $A$ of reals, and every $i<2$, there exist $x<_{W} y$ in $A$ such that $c(x, y)=i$.

In the 1960s, Erdös and his school initiated a systematic study of this sort of colorings, and introduced the following piece of notation. We say that $\lambda \nrightarrow[\mu]_{\theta}^{2}$ holds provided that there exists a symmetric coloring of pairs $d:[\lambda]^{2} \rightarrow \theta$ with the property that for every subset $A$ of $\lambda$ of size $\mu$, and every color $\gamma<\theta$, there exist $\alpha<\beta$ in $A$ such that $d(\alpha, \beta)=\gamma$. So, Sierpiński's partition is a witness to $2^{\aleph_{0}} \nrightarrow\left[\aleph_{1}\right]_{2}^{2}$. Erdös, Hajnal and Rado proved [2] that, assuming the Generalized Continuum Hypothesis (GCH), $\lambda^{+} \nrightarrow\left[\lambda^{+}\right]_{\lambda^{+}}^{2}$ holds for every infinite cardinal $\lambda$. On its face, the existence of such a coloring $d:\left[\lambda^{+}\right]^{2} \rightarrow \lambda^{+}$that attains all possible colors on all squares of unbounded subsets of $\lambda^{+}$appears to be the strongest conceivable failure of Ramsey's theorem at the level of successor cardinals. However, one can ask for more. To see this, let us revisit Sierpiński's example. Let $R$ be a "rectangle" of the form $\left\{(x, y) \in I \times J \mid x<_{W} y\right\}$, for disjoint real intervals $I$ and $J ;^{1}$ then $c \uparrow R$ is a constant function. So, while $c$ admits no monochromatic uncountable squares, it does admit monochromatic uncountable rectangles. In contrast, the Erdős-Hajnal-Rado coloring does attain all colors even on rectangles. Of course, this appears to come with a price: the Erdös-Hajnal-Rado

\footnotetext{
${ }^{1}$ By convention, for sets of ordinals $A, B$, one associates the "rectangle" $A \circledast B=$ $\{(\alpha, \beta) \in A \times B \mid \alpha<\beta\}$.
} 
construction requires the $\mathrm{GCH}$. We refer the reader to $[8,10]$ for a resolution of this particular aspect, and turn now to a further finer concept:

Definition 1.1 (Shelah, [12]). $\operatorname{Pr}_{1}(\lambda, \mu, \theta, \chi)$ asserts the existence of a coloring $d:[\lambda]^{2} \rightarrow \theta$ such that for any family $\mathcal{A} \subseteq[\lambda]^{<\chi}$ of size $\mu$, consisting of pairwise disjoint sets, and every color $\gamma<\theta$, there exist $a, b \in \mathcal{A}$ with $\sup (a)<\min (b)$ satisfying $d[a \times b]=\{\gamma\}$.

So, Sierpiński's example is a coloring satisfying $\operatorname{Pr}_{1}\left(2^{\aleph_{0}}, \aleph_{1}, 2,2\right)$, that fails to satisfy $\operatorname{Pr}_{1}\left(2^{\aleph_{0}}, \aleph_{1}, 2,3\right)$. This justifies the inclusion of the fourth parameter $\chi$. Another justification, and, in fact, the origin of this concept, is in its effect on questions concerning chain conditions of product of topological spaces and related objects. For example, Galvin [4] proved that for $\lambda$ regular uncountable, $\operatorname{Pr}_{1}(\lambda, \lambda, 2, \omega)$ entails two $\lambda$-cc Boolean algebras whose product is not $\lambda$-cc (see also $[9,14]$ ).

Now, what about the third parameter $\theta$ ? Here, we mention that, for example, Shelah proved [16] that $\operatorname{Pr}_{1}\left(\lambda^{+}, \lambda^{+}, \operatorname{cf}(\lambda), \operatorname{cf}(\lambda)\right)$ holds for every singular cardinal $\lambda$, whereas the question of whether $\operatorname{Pr}_{1}\left(\lambda^{+}, \lambda^{+}, \lambda^{+}, \operatorname{cf}(\lambda)\right)$ (or just $\operatorname{Pr}_{1}\left(\lambda^{+}, \lambda^{+}, \lambda, 2\right)$ ) holds for every singular cardinal $\lambda$ is the oldest open problem of this field.

A major breakthrough by Todorčević $[20]$ was proving that $\aleph_{1} \nrightarrow\left[\aleph_{1}\right]_{\aleph_{1}}^{2}$ holds outright in ZFC. Moreover, in the presence of a nonreflecting stationary set, Todorčević's technology generalizes to arbitrary regular cardinals $>\aleph_{1}$, yielding:

Theorem 1.2 (Todorčević, [20]). If $\lambda>\aleph_{1}$ is a regular cardinal that admits a nonreflecting stationary set, then $\lambda \nrightarrow[\lambda]_{\lambda}^{2}$. That is, $\operatorname{Pr}_{1}(\lambda, \lambda, \lambda, \chi)$ holds for $\chi=2$.

This generalization was independently established by Shelah shortly after learning the proof of $\aleph_{1} \nrightarrow\left[\aleph_{1}\right]_{\aleph_{1}}^{2}$, and appeared in [13].

Theorem 1.2 raises the question whether under the same hypothesis, the above holds true also for higher $\chi$ 's?

This particular question and its variations were studied systematically by Shelah in a sequence of papers [11-15, 18, 19], and in his monograph [16]. Roughly speaking, the difficulty in establishing $\operatorname{Pr}_{1}(\lambda, \lambda, \lambda, \chi)$ for $\chi>2$ is the need for some room to either derive several oscillation functions (and then to contrast them), or to enforce repetitions that allow to find for every family $\mathcal{A} \subseteq[\lambda]^{<\chi}$ as in Definition 1.1 , an equipotent subfamily $\mathcal{A}^{\prime} \subseteq \mathcal{A}$ which is less diverse or more tamed in various senses. In [14], this was obtained through the arithmetic assumption " $2^{\chi}<\lambda$ ", and in [15], $\operatorname{Pr}_{1}(\lambda, \lambda, \lambda, \chi)$ 
was established for $\chi=\aleph_{0}$ and a cardinal $\lambda$ that admits a nonreflecting stationary set $S \subseteq \lambda$, through the requirement that for all $\alpha \in S, \operatorname{cf}(\alpha)$ is at least the double-successor of $\chi$. Later on, in [18], it was proved that $\operatorname{Pr}_{1}\left(\chi^{++}, \chi^{++}, \chi^{++}, \chi\right)$ holds for every regular cardinal $\chi$.

In this paper, we eliminate the arithmetic hypothesis from [14], eliminate the "double successor" cofinality gap requirement from [15], obtain the main results of $[18,19]$ as a corollary, and indeed increase $\chi$ from 2 to $\omega$ in Theorem 1.2 above. It is proved:

Main result. If $\lambda, \chi$ are regular cardinal, $\lambda>\chi^{+}$, and $E_{\geq \chi}^{\lambda}$ admits a nonreflecting stationary set, then $\operatorname{Pr}_{1}(\lambda, \lambda, \lambda, \chi)$ holds.

\subsection{About the proof}

In [18], Shelah introduced a coloring principle $\operatorname{Pr}_{6}(\kappa, \kappa, \theta, \chi)$, proved that $\operatorname{Pr}_{6}\left(\mu^{+}, \mu^{+}, \mu^{+}, \mu\right)$ holds for every regular cardinal $\mu$, and provided a lifting theorem:

Theorem 1.3 (Shelah, [18]). If $\operatorname{Pr}_{6}(\kappa, \kappa, \theta, \chi)$ holds, $\chi \leq \kappa<\operatorname{cf}(\lambda)=\lambda$, then $\operatorname{Pr}_{1}(\lambda, \lambda, \theta, \chi)$ holds, provided that there exists a nonreflecting stationary subset of $E_{\geq \kappa}^{\lambda}$.

Unfortunately, if $\theta$ is considerably smaller than $\lambda$, it is unclear how to infer $\operatorname{Pr}_{1}(\lambda, \lambda, \lambda, \chi)$ from $\operatorname{Pr}_{1}(\lambda, \lambda, \theta, \chi){ }^{2}$ So, for instance, it is unclear how to deduce the main result of [15] from the above strategy. Moreover, by Ramsey's theorem, $\operatorname{Pr}_{6}(\kappa, \kappa, \theta, \chi)$ fails for $\kappa=\aleph_{0}$ and $\theta \geq 2$, so if $\lambda$ admits a nonreflecting stationary set, but every stationary subset of $E_{>\omega}^{\lambda}$ reflects, then the above theorem does not come into play.

In this paper, we introduce a relative of $\operatorname{Pr}_{6}$ that overcomes these two barriers. We name it $\mathrm{P} \ell_{6}$, and prove:

Theorem 1.4. If $\mathrm{P}_{6}(\kappa, \chi)$ holds, $\chi \leq \kappa<\operatorname{cf}(\lambda)=\lambda$, then $\operatorname{Pr}_{1}(\lambda, \lambda, \lambda, \chi)$ holds, provided that there exists a nonreflecting stationary subset of $E_{\geq \chi}^{\lambda}$.

This time, the main result of [15] does follow as a corollary, provided that $\mathrm{P} \ell_{6}\left(\aleph_{2}, \aleph_{0}\right)$ (or $\left.\mathrm{P} \ell_{6}\left(\aleph_{1}, \aleph_{0}\right)\right)$ hold. But does $\mathrm{P} \ell_{6}\left(\aleph_{2}, \aleph_{0}\right)$ hold? It does. In fact, it will be proved that $\mathrm{P} \ell_{6}\left(\mu^{+}, \mu\right)$ holds for every regular cardinal $\mu$. Consequently:

\footnotetext{
${ }^{2}$ Recall the problem mentioned earlier concerning successor of singulars.
} 
Corollary 1.5. If $\lambda>\aleph_{1}$ is a regular cardinal that admits a nonreflecting stationary set, then $\operatorname{Pr}_{1}\left(\lambda, \lambda, \lambda, \aleph_{0}\right)$ holds.

The preceding is optimal, since Martin's Axiom, $\mathrm{MA}_{\aleph_{1}}$, entails the failure of $\operatorname{Pr}_{1}\left(\aleph_{1}, \aleph_{1}, 2, \aleph_{0}\right) \cdot{ }^{3}$

\subsection{Conventions}

For a set $a$ of ordinals, denote $\operatorname{acc}^{+}(a):=\{\alpha<\sup (a) \mid \sup (a \cap \alpha)=\alpha\}$, $\operatorname{acc}(a):=a \cap \operatorname{acc}^{+}(a)$ and $\operatorname{nacc}(a):=a \backslash \operatorname{acc}(a)$. For $i<\operatorname{otp}(a)$, we let $a(i)$ denote the unique ordinal $\alpha \in a$ such that $\operatorname{otp}(a \cap \alpha)=i$. The length of a finite sequence $\sigma$ is denoted by $\ell(\sigma)$.

\section{The decomposition principle $\mathrm{P} \ell_{6}$}

This section is dedicated to the study of the following principle.

Definition 2.1. $\mathrm{P} \ell_{6}(\kappa, \chi)$ asserts the existence of a coloring $c:{ }^{<\omega} \kappa \rightarrow \omega$ satisfying the following. For every sequence $\left\langle\left(u_{\alpha}, v_{\alpha}, \rho_{\alpha}\right) \mid \alpha<\kappa\right\rangle$ and $\varphi$ : $\kappa \rightarrow \kappa$ with

(1) $\varphi$ is eventually regressive. That is, $\varphi(\alpha)<\alpha$ for co-boundedly many $\alpha<\kappa$

(2) $u_{\alpha}$ and $v_{\alpha}$ are nonempty elements of $\left[{ }^{<\omega} \kappa\right]^{<\chi}$;

(3) $\alpha \in \operatorname{Im}(\eta)$ for all $\eta \in u_{\alpha}$;

(4) $\rho_{\alpha} \frown\langle\alpha\rangle \sqsubseteq \rho$ for all $\rho \in v_{\alpha}$,

there exist $\alpha<\beta<\kappa$ with $\varphi(\alpha)=\varphi(\beta)$ such that $c\left(\eta^{\frown} \rho\right)=\ell(\eta)$ for all $\eta \in$ $u_{\alpha}$ and $\rho \in v_{\beta}$.

At a first glance, it may seem that $\mathrm{P} \ell_{6}$ puts an impossible task on $c$ : decomposing a concatenated sequence back into its original ingredients. However, the main result of this section reads as follows.

Theorem 2.2. $\mathrm{P} \ell_{6}\left(\mu^{+}, \mu\right)$ holds for every regular cardinal $\mu$.

\footnotetext{
${ }^{3}$ Kunen [6], Rowbottom [5], Solovay, and others (see [3, Section 41M]) proved that $\mathrm{MA}_{\aleph_{1}}$ implies that the product of any two $c c c$ topological spaces is again $c c c$, while Galvin [4] proved that $\operatorname{Pr}_{1}\left(\aleph_{1}, \aleph_{1}, 2, \aleph_{0}\right)$ entails two $c c c$ topological spaces $\mathbb{X}, \mathbb{Y}$ for which $\mathbb{X} \times \mathbb{Y}$ is not $c c c$.
} 
Theorem 2.2 is obtained as an immediate corollary to Theorems 2.3 and 2.6 below, which are somewhat more informative.

Theorem 2.3. Suppose that $\mu$ is a regular cardinal, and there exists a cardinal $\theta<\mu$ with $2^{\theta} \geq \mu$. Then the following strong form of $\mathrm{P} \ell_{6}\left(\mu^{+}, \mu\right)$ holds. Write $\Gamma:=E_{\mu}^{\mu^{+}}$and $\kappa:=\mu^{+}$. There exists a coloring $c:<\omega_{\kappa} \rightarrow \omega \times$ $\kappa \times \kappa \times \kappa$, such that for every $\gamma^{*}<\kappa$, a sequence $\left\langle\left(u_{\alpha}, v_{\alpha}, \rho_{\alpha}\right) \mid \alpha \in \Gamma\right\rangle$, and $\varphi: \Gamma \rightarrow \kappa$ satisfying clauses (1)-(4) of Definition 2.1, there exist $\alpha<\beta$ in $\Gamma$ with $\varphi(\alpha)=\varphi(\beta)$ such that $c(\eta \frown \rho)=\left(\ell(\eta), \alpha, \beta, \gamma^{*}\right)$ for all $\eta \in u_{\alpha}$ and $\rho \in v_{\beta}$.

Proof. We commence with the construction of the coloring, and then verify that this works.

The coloring. Let $\vec{C}=\left\langle C_{\delta} \mid \delta<\kappa\right\rangle$ be a sequence such that $C_{\delta}$ is a club in $\delta$ of minimal order-type for every limit $\delta<\kappa$, and such that $C_{\delta+1}=\{\delta\}$ for all $\delta<\kappa$. By [18, Claim 3.1], [18, Lemma 3.10] and the existence of $\theta<\mu$ with $2^{\theta} \geq \mu$, we may moveover require that $\vec{C} \uparrow \Gamma$ enjoys the following form of club guessing. ${ }^{4}$ There exist $\left\langle g_{\delta}: C_{\delta} \rightarrow \omega \mid \delta \in \Gamma\right\rangle$ and $\psi: \kappa \rightarrow \kappa$ with the property that for every club $D \subseteq \kappa$, and every $\gamma^{*}<\kappa$, there exists some $\delta \in \Gamma$ with $\psi(\delta)=\gamma^{*}$ such that $\sup \left\{\zeta \in \operatorname{nacc}\left(C_{\delta}\right) \cap D \mid g_{\delta}(\zeta)=n\right\}=\delta$ for all $n<\omega$.

Fix a coloring $d:[\kappa]^{2} \rightarrow \omega$ that satisfies $d(\alpha, \beta)=g_{\beta}\left(\min \left(C_{\beta} \backslash \alpha\right)\right)$ for all $\alpha \in \beta \in \Gamma$.

Next, given a sequence $\sigma \in \epsilon^{<\omega} \kappa$, let

$$
\mathcal{D}_{\sigma}:=\{(i, j) \mid i<j<\ell(\sigma) \& \sigma(i)<\sigma(j)\},
$$

and whenever $\mathcal{D}_{\sigma} \neq \emptyset$, set

- $\mathfrak{m}_{\sigma}:=\max \left\{\operatorname{otp}\left(C_{\sigma(j)} \cap \sigma(i)\right) \mid(i, j) \in \mathcal{D}_{\sigma}\right\}$;

- $\mathcal{P}_{\sigma}:=\left\{(i, j) \in \mathcal{D}_{\sigma} \mid \operatorname{otp}\left(C_{\sigma(j)} \cap \sigma(i)\right)=\mathfrak{m}_{\sigma}\right\}$;

- $j_{\sigma}:=\min \left\{j \mid \exists i(i, j) \in \mathcal{P}_{\sigma}\right\}$;

- $\alpha_{\sigma}:=\min \left\{\sigma(i) \mid \exists j(i, j) \in \mathcal{P}_{\sigma}\right\}$;

- $\beta_{\sigma}:=\sigma\left(j_{\sigma}\right)$.

Define $c_{i}:{ }^{<\omega} \kappa \rightarrow \kappa$ for $i<4$ so that for every $\sigma \in{ }^{<\omega} \kappa$ with $\mathcal{D}_{\sigma} \neq \emptyset$ :

- $c_{0}(\sigma)=\max \left\{0, j_{\sigma}-d\left(\alpha_{\sigma}, \beta_{\sigma}\right)\right\}$;

\footnotetext{
${ }^{4}$ This is the only application of the arithmetic hypothesis in the whole proof.
} 
- $c_{1}(\sigma):=\alpha_{\sigma}$;

- $c_{2}(\sigma):=\beta_{\sigma}$;

- $c_{3}(\sigma):=\psi\left(\beta_{\sigma}\right)$.

We claim that $\sigma \mapsto\left(c_{0}(\sigma), c_{1}(\sigma), c_{2}(\sigma), c_{3}(\sigma)\right)$ works.

Verification. Suppose that we are given a sequence $\left\langle\left(u_{\alpha}, v_{\alpha}, \rho_{\alpha}\right) \mid \alpha \in \Gamma\right\rangle$ together with a prescribed color $\gamma^{*}<\kappa$, and a map $\varphi: \Gamma \rightarrow \kappa$, as in the statement of the theorem. Note that, without loss of generality, we may assume that $\alpha \notin \operatorname{Im}\left(\rho_{\alpha}\right)$ for all $\alpha \in \Gamma$. Indeed, we may assume that $\rho_{\alpha}$ denotes the shortest possible sequence to satisfy $\rho_{\alpha} \frown\langle\alpha\rangle \sqsubseteq \rho$ for all $\rho \in v_{\alpha}$.

Let $\lambda$ be a large enough regular cardinal, and let $\unlhd_{\lambda}$ be a well-ordering of $\mathcal{H}_{\lambda}$. Let $\left\langle M_{\delta} \mid \delta<\kappa\right\rangle$ be a continuous $\in$-chain of elementary submodels of $\left(\mathcal{H}_{\lambda}, \in, \unlhd_{\lambda}\right)$, each of size $\mu$, such that $\mu \subseteq M_{0}$ and $\left\{\left\langle C_{\delta} \mid \delta \in \Gamma\right\rangle,\left\langle\left(u_{\alpha}, v_{\alpha}, \rho_{\alpha}\right)\right|\right.$ $\alpha \in \Gamma\rangle, \varphi\} \in M_{0}$.

Write $D:=\left\{\delta<\kappa \mid M_{\delta} \cap \kappa=\delta\right\}$. By the club-guessing feature of $\left\langle C_{\delta}\right|$ $\delta \in \Gamma\rangle$, let us pick $\beta \in \Gamma$ with $\psi(\beta)=\gamma^{*}$ such that $\sup \left\{\zeta \in \operatorname{nacc}\left(C_{\beta}\right) \cap D \mid\right.$ $\left.g_{\beta}(\zeta)=n\right\}=\beta$ for all $n<\omega$. In particular, $\beta \in D$ and $\varphi(\beta)<\beta$. We shall find $\alpha \in \beta \cap \Gamma$ with $\varphi(\alpha)=\varphi(\beta)$ such that $c_{0}(\eta \frown \rho)=\ell(\eta), c_{1}(\eta \frown \rho)=\alpha$, $c_{2}(\eta \frown \rho)=\beta$ and $c_{3}(\eta \frown \rho)=\gamma^{*}$ for all $\eta \in u_{\alpha}$ and $\rho \in v_{\beta}$.

Define $h:[\kappa]^{<\mu} \rightarrow \mu$ by stipulating

$$
h(z):=\sup \left\{\operatorname{otp}\left(C_{\beta^{\prime}} \cap \alpha^{\prime}\right) \mid \alpha^{\prime}<\beta^{\prime} \text { are in } z\right\} .
$$

For every $\delta \in \Gamma$, write $a_{\delta}:=\bigcup\left\{\operatorname{Im}(\sigma) \mid \sigma \in u_{\delta} \cup v_{\delta}\right\}$, and $x_{\delta}:=a_{\delta} \backslash \delta$.

Put $\xi:=\sup \left(a_{\beta} \cap \beta\right)+1$. As $\left|a_{\beta}\right|<\operatorname{cf}(\beta)$, we have $\xi<\beta$. Let $f: \mu \rightarrow$ $\xi$ be the $\unlhd_{\lambda}$-least surjection. By $\left|a_{\beta}\right|<\mu$ and regularity of the latter, let $i^{\prime}<\mu$ be large enough so that $a_{\beta} \cap \beta \subseteq f\left[i^{\prime}\right]$. Write $n^{*}:=\ell\left(\rho_{\beta}\right), z:=f\left[i^{\prime}\right]$, $\epsilon:=h\left(a_{\beta} \cup z\right), \tau:=\varphi(\beta)$, and

$$
T:=\left\{\delta \in \Gamma \mid a_{\delta} \cap \delta \subseteq z, h\left(a_{\delta} \cup z\right)=\epsilon, \varphi(\delta)=\tau\right\} .
$$

Pick a large enough $\zeta \in \operatorname{nacc}\left(C_{\beta}\right) \cap D$ above $\max \{\xi, \tau\}$ such that $g_{\beta}(\zeta)=$ $n^{*}$, and $\operatorname{otp}\left(C_{\beta} \cap \zeta\right)>\epsilon$. By $\xi, \tau \in M_{\zeta}$, we have $T \in M_{\zeta}$. Since $\beta \in T \backslash M_{\zeta}$, we have $\sup \left(T \cap M_{\zeta}\right)=\zeta$. So, pick a large enough $\alpha \in T \cap M_{\zeta}$ such that $\alpha>\max \left(C_{\beta} \cap \zeta\right)$. In particular, $\varphi(\alpha)=\varphi(\beta)$.

Claim 2.3.1. (1) $h\left(a_{\alpha} \cup z\right)=\epsilon$;

(2) $a_{\alpha} \cap \alpha \subseteq z$;

(3) $x_{\alpha} \subseteq\left(\max \left(C_{\beta} \cap \zeta\right), \zeta\right)$. In particular, otp $\left(C_{\beta} \cap \alpha\right)>\epsilon$, and 
(4) $d(\alpha, \beta)=\ell\left(\rho_{\beta}\right)$.

Proof. (1) and (2) are consequences of the fact that $\alpha$ is a member of $T$.

(3) By $\alpha \in M_{\zeta}$, we have $x_{\alpha} \in M_{\zeta}$, and then $\sup \left(x_{\alpha}\right)<\zeta$. By $\alpha \in \operatorname{Im}(\eta)$ for all $\eta \in u_{\alpha}$, we have $\min \left(x_{\alpha}\right)=\alpha$. Altogether, $\max \left(C_{\beta} \cap \zeta\right)<\alpha=\min \left(x_{\alpha}\right)$ $\leq \sup \left(x_{\alpha}\right)<\zeta$. In particular, otp $\left(C_{\beta} \cap \alpha\right)=\operatorname{otp}\left(C_{\beta} \cap \zeta\right)>\epsilon$.

(4) By the previous clause, and the choice of $d$, we have $d(\alpha, \beta)=g_{\beta}(\zeta)=$ $n^{*}=\ell\left(\rho_{\beta}\right)$.

To see that $\alpha, \beta$ are as desired, suppose that we are given $\eta \in u_{\alpha}$ and $\rho \in v_{\beta}$, and let us show that $c_{0}(\sigma)=\ell(\eta), c_{1}(\sigma)=\alpha, c_{2}(\sigma)=\beta$ and $c_{3}(\sigma)=\gamma^{*}$, for $\sigma:=\eta \frown \rho$.

By $\alpha \in \operatorname{Im}(\eta)$ and $\beta \in \operatorname{Im}(\rho)$, there exist $\hat{i}<\hat{j}<\ell(\sigma)$ such that $\sigma(\hat{i})=\alpha$ and $\sigma(\hat{j})=\beta$. So $(\hat{i}, \hat{j})$ witnesses that $\mathcal{D}_{\sigma} \neq \emptyset$, and then by Claim 2.3.1(3), we have $\mathfrak{m}_{\sigma} \geq \operatorname{otp}\left(C_{\beta} \cap \alpha\right)>\epsilon$.

Claim 2.3.2. If $(i, j) \in \mathcal{P}_{\sigma}$, then:

(1) $\{\sigma(i), \sigma(j)\} \nsubseteq\left(a_{\alpha} \cup z\right)$, and $\{\sigma(i), \sigma(j)\} \nsubseteq\left(a_{\beta} \cup z\right)$;

(2) If $\sigma(i) \in a_{\alpha}$, then $\sigma(j) \notin a_{\beta} \cap \beta$;

(3) $\eta \sqsubseteq \sigma \uparrow j$;

(4) $\sigma(j)=\beta$;

(5) $\sigma(i) \in x_{\alpha}$.

Proof. (1) If $\{\sigma(i), \sigma(j)\} \subseteq a_{\alpha} \cup z$, then $\operatorname{otp}\left(C_{\sigma(j)} \cap \sigma(i)\right) \leq h\left(a_{\alpha} \cup z\right)=\epsilon$, by Claim 2.3.1(1). Likewise, if $\{\sigma(i), \sigma(j)\} \subseteq a_{\beta} \cup z$, then $\operatorname{otp}\left(C_{\sigma(j)} \cap\right.$ $\sigma(i)) \leq h\left(a_{\beta} \cup z\right)=\epsilon$, by definition of $\epsilon$. However, otp $\left(C_{\sigma(j)} \cap \sigma(i)\right)=$ $\mathfrak{m}_{\sigma}>\epsilon$.

(2) Otherwise, $\{\sigma(i), \sigma(j)\} \subseteq a_{\alpha} \cup\left(a_{\beta} \cap \beta\right) \subseteq a_{\alpha} \cup z$, contradicting the previous clause.

(3) By $\sigma=\eta \frown \rho$, we have $\{\sigma(i), \sigma(j)\} \subseteq \operatorname{Im}(\eta) \cup \operatorname{Im}(\rho) \subseteq a_{\alpha} \cup a_{\beta}$. So, by Clause (1), together with $i<j$ and $\sigma=\eta \frown \rho$, we infer that $\sigma(i) \in$ $\operatorname{Im}(\eta)$ and $\sigma(j) \in \operatorname{Im}(\rho)$. That is, $i<\ell(\eta)$ and $j \geq \ell(\eta)$. In particular, $\sigma(i) \in a_{\alpha}$ and $\sigma(j) \in a_{\beta}$.

(4) By $\sigma(i) \in a_{\alpha}, \sigma(j) \in a_{\beta}$, and Clause (2), we have $\sigma(j) \geq \beta$. Towards a contradiction, suppose that $\beta<\sigma(j)$. Then by $\sigma(i) \in a_{\alpha}$ and $\alpha \in \beta \in$ $D$, we have $\sigma(i)<\beta<\sigma(j)$, and hence otp $\left(C_{\sigma(j)} \cap \sigma(i)\right) \leq \operatorname{otp}\left(C_{\sigma(j)} \cap\right.$ 
$\beta)$. As $\sigma(j), \beta \in a_{\beta}$, we then get that $\operatorname{otp}\left(C_{\sigma(j)} \cap \sigma(i)\right) \leq h\left(a_{\beta}\right) \leq \epsilon$, contradicting the fact that $\operatorname{otp}\left(C_{\sigma(j)} \cap \sigma(i)\right)=\mathfrak{m}_{\sigma}>\epsilon$.

(5) Otherwise, $\sigma(i) \in a_{\alpha} \cap \alpha \subseteq z$ and $\{\sigma(i), \sigma(j)\} \subseteq\left(z \cup a_{\beta}\right)$, contradicting Clause (1).

As $\beta \notin \operatorname{Im}\left(\rho_{\beta}\right)$, we get from the preceding claim, and the minimality of $j_{\sigma}$, that

$$
\sigma \uparrow\left(j_{\sigma}+1\right)=\eta \frown \rho_{\beta} \frown\langle\beta\rangle .
$$

So $\beta_{\sigma}=\beta$ and $j_{\sigma}=\ell\left(\eta \frown \rho_{\beta}\right)$. By Clause (5) of the preceding claim, $\alpha_{\sigma} \in x_{\alpha}$. Then, by Claim 2.3.1(3), we get that $\operatorname{otp}\left(C_{\beta} \cap \alpha_{\sigma}\right)=\operatorname{otp}\left(C_{\beta} \cap \alpha\right)$. Recalling that $\min \left(x_{\alpha}\right)=\alpha \in \operatorname{Im}(\eta)$, Claims 2.3.2(5) and 2.3.1(3), then imply that $\alpha_{\sigma}=\alpha$.

Altogether, by Claim 2.3.1(4):

- $\sigma \uparrow\left(j_{\sigma}-d\left(\alpha_{\sigma}, \beta_{\sigma}\right)\right)=(\eta \frown \rho) \uparrow\left(\ell\left(\eta \frown \rho_{\beta}\right)-\ell\left(\rho_{\beta}\right)\right)=\eta$;

- $c_{0}(\sigma)=\max \left\{0, j_{\sigma}-d\left(\alpha_{\sigma}, \beta_{\sigma}\right)\right\}=\ell(\eta)$;

- $c_{1}(\sigma)=\alpha_{\sigma}=\alpha$

- $c_{2}(\sigma)=\beta_{\sigma}=\beta$;

- $c_{3}(\sigma)=\psi\left(\beta_{\sigma}\right)=\gamma^{*}$.

Our goal now is to handle the other case: $\mu$ strongly inaccessible (including $\mu=\aleph_{0}$ ). For this, we first need to state and prove a straightforward generalization of a fact from [20].

Theorem 2.4 (Todorčević, implicit in [20]). If $\mu^{<\mu}=\mu$, then there exists a coloring $d:\left[\mu^{+}\right]^{2} \rightarrow \mu^{+}$satisfying the following.

For every family $\mathcal{A} \subseteq \mathcal{P}\left(\mu^{+}\right)$of size $\mu^{+}$consisting of pairwise disjoint sets of some fixed order-type $\chi<\mu$, and every color $\gamma^{*}<\mu^{+}$, there exist $\mathcal{A}^{\prime} \in[\mathcal{A}]^{\mu}$ and $b \in \mathcal{A}$ such that $\sup (a)<\min (b)$ and $d(a(i), b(i))=\gamma^{*}$ for all $a \in \mathcal{A}^{\prime}$ and $i<\chi$.

Proof. Let $\left\langle e_{\beta}: \beta \rightarrow \mu \mid \beta<\mu^{+}\right\rangle$be a sequence of injections. Let $\left\langle f_{\alpha}: \mu \rightarrow\right.$ $\mu\left|\alpha<\mu^{+}\right\rangle$be a sequence of pairwise distinct functions. Write $\Delta(\alpha, \alpha):=$ $\infty$, and $\Delta(\alpha, \beta):=\min \left\{i<\mu \mid f_{\alpha}(i) \neq f_{\beta}(i)\right\}$ for distinct $\alpha, \beta<\mu^{+}$. Put $\Gamma:=E_{\mu}^{\mu^{+}}$. Fix a surjection $\psi: \mu^{+} \rightarrow \mu^{+}$such that $\Gamma_{\gamma}:=\{\delta \in \Gamma \mid \psi(\delta)=\gamma\}$ is stationary for every $\gamma<\mu^{+}$. Define a coloring $c:\left[\mu^{+}\right]^{2} \rightarrow \mu^{+}$by letting 
for all $\alpha<\beta<\mu^{+}$:

$$
c(\alpha, \beta):=\min \left(\left\{\delta \in[\alpha, \beta) \mid \Delta(\alpha, \beta) \geq e_{\beta}(\delta)\right\} \cup\{\beta\}\right)
$$

Finally, let $d(\alpha, \beta):=\psi(c(\alpha, \beta))$.

To see that $d$ works, suppose that $\mathcal{A}, \chi$ and $\gamma^{*}$ are as in the hypothesis. Let $\mathcal{M}$ be an elementary submodel of $\left(\mathcal{H}_{\lambda}, \in, \triangleleft_{\lambda}\right)$ for a large enough regular cardinal $\lambda$, of size $\mu$, such that $\left\{\mathcal{A},\left\langle e_{\alpha} \mid \alpha<\mu^{+}\right\rangle,\left\langle f_{\alpha} \mid \alpha<\mu^{+}\right\rangle,{ }^{<\mu} \mu\right\} \in \mathcal{M}$, and $\mathcal{M} \cap \mu^{+} \in \Gamma_{\gamma^{*}}$. Denote $\delta:=\mathcal{M} \cap \mu^{+}$. Pick $b \in \mathcal{A}$ with $\min (b)>\delta$. Our goal now is to find an $\mathcal{A}^{\prime} \in[\mathcal{A} \cap \mathcal{M}]^{\mu}$ such that $c(a(i), b(i))=\delta$ for all $a \in \mathcal{A}^{\prime}$ and $i<\chi$.

Put

- $\epsilon:=\sup \left\{e_{b(i)}(\delta) \mid i<\chi\right\}$

- $t:=\left\langle f_{b(i)} \uparrow \epsilon \mid i<\chi\right\rangle$.

By $<\mu \mu \in \mathcal{M}$ and $\left.\right|^{<\mu} \mu|=| \mathcal{M} \mid$, we have $t \in \mathcal{M}$, and hence the set

$$
\mathcal{A}_{t}:=\left\{a \in \mathcal{A} \mid t=\left\langle f_{a(i)}\lceil\epsilon|i<\chi\rangle\}\right.\right.
$$

belongs to $\mathcal{M}$. Since $b \in \mathcal{A}_{t} \backslash \mathcal{M}$, we get that $\left|\mathcal{A}_{t}\right|=\mu^{+}$. Pick $x \in \mathcal{A}_{t} \backslash(\mathcal{M} \cup$ $\{b\})$. Put

- $\nu:=\sup \{\Delta(b(i), x(i)) \mid i<\chi\}$

- $s:=\left\langle f_{x(i)} \uparrow(\nu+1) \mid i<\chi\right\rangle$;

- $D:=\bigcup_{i<\chi}\left\{\delta^{\prime}<\delta \mid e_{b(i)}\left(\delta^{\prime}\right) \leq \nu\right\}$.

As $e_{b(i)}$ is injective for all $i<\chi$, and $\mu$ is regular, $|D|<\mu$. Since $x \in \mathcal{A}_{s} \mid$ $\mathcal{M}$, we get by elementarity that for every $\tau<\delta$, there exists $a \in \mathcal{A}_{s} \cap \mathcal{M}$ with $\min (a)>\tau$. Put $\mathcal{A}^{\prime}:=\left\{a \in \mathcal{A}_{s} \cap \mathcal{M} \mid \min (a)>\sup (D)\right\}$. $\operatorname{By} \operatorname{cf}(\delta)=\mu$, we have $\left|\mathcal{A}^{\prime}\right|=\mu$.

Finally, to see that $c(a(i), b(i))=\delta$ for all $a \in \mathcal{A}^{\prime}$ and $i<\chi$, fix an arbitrary $a \in \mathcal{A}^{\prime}$ and $i<\chi$. We have:

- $t(i)=f_{b(i)} \uparrow \epsilon$ by definition of $t$;

- $t(i)=f_{x(i)} \uparrow \epsilon$, since $x \in \mathcal{A}_{t}$;

- $\epsilon \leq \Delta(b(i), x(i))$, by the above two;

- $s(i)=f_{x(i)} \uparrow(\nu+1)$ by definition of $s$;

- $s(i)=f_{a(i)} \uparrow(\nu+1)$, since $a \in \mathcal{A}_{s}$; 
- $\nu<\Delta(a(i), x(i))$ by the above two;

- $\epsilon \leq \Delta(b(i), x(i)) \leq \nu<\Delta(a(i), x(i))$, and hence $\epsilon \leq \Delta(a(i), b(i)) \leq \nu$;

- $\Delta(a(i), b(i)) \geq \epsilon \geq e_{b(i)}(\delta)$, by definition of $\epsilon$.

It follows that if $c(a(i), b(i)) \neq \delta$, then there must exist some $\delta^{\prime} \in[a(i), \delta)$ such that $\Delta(a(i), b(i)) \geq e_{b(i)}\left(\delta^{\prime}\right)$. But then, $\nu \geq e_{b(i)}\left(\delta^{\prime}\right)$, meaning that $\delta^{\prime} \in$ $D$, and contradicting the fact that $a(i) \geq \min (a)>\sup (D)$.

So, $c(a(i), b(i))=\delta$ and $d(a(i), b(i))=\gamma^{*}$, as desired.

We shall also need a simple lemma.

Lemma 2.5 (folklore). For every infinite cardinal $\mu$, there exists a function $h:\left[\mu^{+}\right]^{2} \rightarrow \mu$ such that:

(1) $h$ is locally small, that is, $|\{\alpha<\beta \mid h(\alpha, \beta) \leq \epsilon\}|<\mu$ for all $(\epsilon, \beta) \in$ $\mu \times \mu^{+}$;

(2) $h$ is transitive, that is, $h(\alpha, \gamma) \leq \max \{h(\alpha, \beta), h(\beta, \gamma)\}$ for all $\alpha<\beta<$ $\gamma<\mu^{+}$.

Proof. For every $\gamma<\mu^{+}$, fix a surjection $\varphi_{\gamma}: \mu \rightarrow \gamma$. Put $A_{0}^{i}:=\emptyset$ for all $i<\mu$. Now, suppose that $\gamma<\mu^{+}$is an ordinal such that for all $\delta<\gamma$ and $i<\mu$, we have $\left|A_{\delta}^{i}\right| \leq|i|+\aleph_{0}$, and $\left\{A_{\delta}^{i} \mid i<\mu\right\}$ is a chain converging to $\delta$. For all $i<\mu$, put:

$$
A_{\gamma}^{i}:=\varphi_{\gamma}[i] \cup \bigcup\left\{A_{\delta}^{i} \mid \delta \in \varphi_{\gamma}[i]\right\} .
$$

Clearly, $\left|A_{\gamma}^{i}\right| \leq|i|+\aleph_{0}$, and $\left\{A_{\gamma}^{i} \mid i<\mu\right\}$ is a chain converging to $\gamma$.

Then, define $h:\left[\mu^{+}\right]^{2} \rightarrow \mu$ by letting for all $\alpha<\beta<\mu^{+}$:

$$
h(\alpha, \beta):=\min \left\{i<\mu \mid \alpha \in A_{\beta}^{i}\right\} .
$$

As $\{\alpha<\beta \mid h(\alpha, \beta) \leq \epsilon\}=A_{\beta}^{\epsilon}$ for all $\epsilon<\mu$, the function $h$ is locally small.

We now prove that $h(\alpha, \gamma) \leq \max \{h(\alpha, \beta), h(\beta, \gamma)\}$ holds for all $\alpha<\beta<$ $\gamma<\mu^{+}$by induction on $\gamma<\mu^{+}$.

Suppose that $\gamma<\mu^{+}$and that transitivity holds for all $\gamma^{\prime}<\gamma$. Let $\alpha<$ $\beta<\gamma$ be arbitrary. Write $i:=\max \{h(\alpha, \beta), h(\beta, \gamma)\}$. By $\beta \in A_{\gamma}^{i}$, there are two cases to consider:

- If $\beta \in \varphi_{\gamma}[i]$, then by definition of $A_{\gamma}^{i}$, we have $A_{\beta}^{i} \subseteq A_{\gamma}^{i}$. So, by $\alpha \in A_{\beta}^{i}$, we have $\alpha \in A_{\gamma}^{i}$, and hence $h(\alpha, \gamma) \leq i$. 
- If $\beta \in A_{\delta}^{i}$ for some $\delta \in \varphi_{\gamma}[i]$, then $h(\beta, \delta) \leq i$, and by the inductive hypothesis, $h(\alpha, \delta) \leq \max \{h(\alpha, \beta), h(\beta, \delta)\} \leq i$. So, $\alpha \in A_{\delta}^{i}$, and hence $\alpha \in A_{\gamma}^{i}$. That is, $h(\alpha, \gamma) \leq i$.

Theorem 2.6. If $\mu^{<\mu}=\mu$, then $\mathrm{P} \ell_{6}\left(\mu^{+}, \mu\right)$ holds in the following strong sense.

Letting $\kappa:=\mu^{+}$, there exists a coloring $c:{ }^{<\omega} \kappa \rightarrow \omega \times \kappa \times \kappa \times \kappa$, such that for every $\gamma^{*}<\kappa$, and every sequence $\left\langle\left(u_{\alpha}, v_{\alpha}, \rho_{\alpha}\right) \mid \alpha \in \Gamma\right\rangle$, with

(1) $\Gamma$ is an unbounded subset of $\kappa$;

(2) $u_{\alpha}$ and $v_{\alpha}$ are nonempty elements of $\left[{ }^{<\omega} \kappa\right]^{<\mu}$;

(3) $\alpha \in \operatorname{Im}(\eta)$ for all $\eta \in u_{\alpha}$;

(4) $\rho_{\alpha} \frown\langle\alpha\rangle \sqsubseteq \rho$ for all $\rho \in v_{\alpha}$,

there exist $\alpha<\beta$ in $\Gamma$ satisfying $c(\eta \frown \rho)=\left(\ell(\eta), \alpha, \beta, \gamma^{*}\right)$ for all $\eta \in u_{\alpha}$ and $\rho \in v_{\beta}$.

Proof. We commence with the construction of the coloring, and then verify that this works.

The coloring. Let $\left\langle f_{\alpha}: \mu \rightarrow 2 \mid \alpha<\mu^{+}\right\rangle$be a sequence of pairwise distinct functions. Write $\Delta(\alpha, \alpha):=\infty$, and $\Delta(\alpha, \beta):=\min \left\{i<\mu \mid f_{\alpha}(i) \neq f_{\beta}(i)\right\}$ for distinct $\alpha, \beta<\mu^{+}$. Let $h:\left[\mu^{+}\right]^{2} \rightarrow \mu$ be transitive and locally small. For every nonconstant sequence $\sigma \in{ }^{<\omega} \kappa$, let

- $\mathcal{D}_{\sigma}:=\left\{(i, j) \mid i<j<\ell(\sigma) \& \forall j^{*}<j\left(\sigma\left(j^{*}\right)<\sigma(j)\right)\right\}$;

- $\Delta_{\sigma}:=\max \{\Delta(\sigma(i), \sigma(j)) \mid i<j<\ell(\sigma) \& \sigma(i) \neq \sigma(j)\}$;

- $\mathfrak{m}_{\sigma}:=\max \left\{h(\sigma(i), \sigma(j)) \mid(i, j) \in \mathcal{D}_{\sigma}\right\}$, provided that $\mathcal{D}_{\sigma} \neq \emptyset$.

- $\mathcal{P}_{\sigma}:=\left\{(i, j) \in \mathcal{D}_{\sigma} \mid h(\sigma(i), \sigma(j))=\mathfrak{m}_{\sigma}\right\}$, provided that $\mathcal{D}_{\sigma} \neq \emptyset$.

- $j_{\sigma}:=\min \left\{j \mid \exists i \quad(i, j) \in \mathcal{P}_{\sigma}\right\}$, provided that $\mathcal{D}_{\sigma} \neq \emptyset$. Otherwise, let $j_{\sigma}:=0$.

By $\mu^{<\mu}=\mu$, and [1], let us fix a sequence $\left\langle g_{\tau}:<\omega \mu^{+} \rightarrow \omega \mid \tau<\mu\right\rangle$ with the property that for every $u \in\left[{ }^{<\omega} \mu^{+}\right]^{<\mu}$ and $g: u \rightarrow \omega$, one can find $\tau<\mu$ with $g=g_{\tau} \uparrow u$.

Let $d:[\kappa]^{2} \rightarrow \kappa$ be given by Theorem 2.4. Let $\psi: \kappa \leftrightarrow \omega \times \mu \times \kappa$ be a bijection. We derive functions $d_{0}:{ }^{<\omega} \kappa \rightarrow \omega, d_{1}:{ }^{<\omega} \kappa \rightarrow \mu$, and $d_{2}:{ }^{<\omega} \kappa \rightarrow$ $\kappa$, as follows. 
Given a nonconstant sequence $\sigma \in{ }^{<\omega} \kappa$, we find the least (say, in lexicographic order) pair $(i, j)$ such that $\Delta(\sigma(i), \sigma(j))=\Delta_{\sigma}$, and let $d_{0}(\sigma):=$ $n, d_{1}(\sigma)=\tau$, and $d_{2}(\sigma):=\gamma$, for the unique $(n, \tau, \gamma)$ such that $\psi(d(\sigma(i)$, $\sigma(j)))=(n, \tau, \gamma)$.

Define $c_{0}:{ }^{<\omega} \kappa \rightarrow{ }^{<\omega} \kappa$ by letting for every nonconstant $\sigma$ :

$$
c_{0}(\sigma):= \begin{cases}\sigma \uparrow\left(j_{\sigma}-d_{0}(\sigma)\right), & j_{\sigma}>d_{0}(\sigma) \\ \emptyset, & \text { otherwise }\end{cases}
$$

Define $c_{i}:{ }^{<\omega} \kappa \rightarrow \kappa$ for $i \in\{1,2,3\}$ by letting for every nonconstant $\sigma$ :

- $c_{1}(\sigma)=\sigma\left(g_{d_{1}(\sigma)}\left(c_{0}(\sigma)\right)\right.$, provided that $g_{d_{1}(\sigma)}\left(c_{0}(\sigma)\right)<\ell(\sigma)$. Otherwise, set $c_{1}(\sigma):=0$;

- $c_{2}(\sigma):=\rho\left(g_{d_{1}(\sigma)}(\rho)\right)$, provided that $\sigma=c_{0}(\sigma) \frown \rho$, and $g_{d_{1}(\sigma)}(\rho)<\ell(\rho)$. Otherwise, set $c_{2}(\sigma):=0$;

- $c_{3}(\sigma):=d_{2}(\sigma)$.

We claim that $\sigma \mapsto\left(\ell\left(c_{0}(\sigma)\right), c_{1}(\sigma), c_{2}(\sigma), c_{3}(\sigma)\right)$ works.

Verification. Suppose that $\Gamma$ is an unbounded subset of $\kappa$, and $\left\langle\left(u_{\alpha}, v_{\alpha}, \rho_{\alpha}\right) \mid \alpha \in \Gamma\right\rangle$ together with a prescribed color $\gamma^{*}<\kappa$ are given as in the statement of the theorem. We shall find $\alpha<\beta$ in $\Gamma$ such that $c_{0}\left(\eta^{-} \rho\right)=$ $\eta, \quad c_{1}(\eta \frown \rho)=\alpha, \quad c_{2}\left(\eta^{\frown} \rho\right)=\beta$ and $\quad c_{3}(\eta \frown \rho)=\gamma^{*}$ for all $\eta \in u_{\alpha}$ and $\rho \in v_{\beta}$.

For every $\delta \in \Gamma$, write $a_{\delta}:=\bigcup\left\{\operatorname{Im}(\sigma) \mid \sigma \in u_{\delta} \cup v_{\delta}\right\}$. By $\mu^{<\mu}=\mu$ and a standard thinning-out procedure, we may assume the existence of $\chi, \chi^{\prime}, \epsilon, t, z$, $n^{*}, \tau^{*}$ such that for all $\delta \in \Gamma$ :

(a) $\operatorname{otp}\left(a_{\delta}\right)=\chi$, and $a_{\delta}\left(\chi^{\prime}\right)=\delta$;

(b) $\sup \left(\Delta "\left[a_{\delta}\right]^{2} \cup h^{\prime \prime}\left[a_{\delta}\right]^{2}\right)=\epsilon$;

(c) $\left\langle f_{a_{\delta}(i)} \uparrow(\epsilon+1) \mid i<\chi\right\rangle=t$;

(d) $\left\{a_{\delta} \mid \delta \in \Gamma\right\}$ forms a $\Delta$-system with root $z ; \sup (z)<\min \left(a_{\alpha} \backslash z\right) \leq$ $\sup \left(a_{\alpha}\right)<\min \left(a_{\delta} \backslash z\right)$ for all $\alpha<\delta$ in $\Gamma$;

(e) $n^{*}=\min \left\{n \mid\left(\rho_{\delta} \frown\langle\delta\rangle\right)(n)>\sup (z)\right\}$;

(f) $\sigma\left(g_{\tau^{*}}(\sigma)\right)=\delta$ for all $\sigma \in u_{\delta} \cup v_{\delta}$.

Claim 2.6.1. There exist $\alpha<\beta$ in $\Gamma$ such that for all $\eta \in u_{\alpha}$ and $\rho \in v_{\beta}$ :

(1) $\mathcal{D}_{\eta-\rho} \neq \emptyset$; 
(2) $\mathfrak{m}_{\eta \frown \rho}>\epsilon$;

(3) $d_{0}(\eta \frown \rho)=n^{*}, d_{1}(\eta \frown \rho)=\tau^{*}$, and $d_{2}(\eta \frown \rho)=\gamma^{*}$.

Proof. Put $\mathcal{A}:=\left\{a_{\delta} \backslash z \mid \delta \in \Gamma \backslash z\right\}$. By the choice of $d$, we now find $\mathcal{A}^{\prime} \in$ $[\mathcal{A}]^{\mu}$ and $b \in \mathcal{A}$ such that $\sup (a)<\min (b)$ and $d(a(i), b(i))=\psi^{-1}\left(n^{*}, \tau^{*}, \gamma^{*}\right)$ for all $a \in \mathcal{A}^{\prime}$ and $i<\operatorname{otp}(a)$. Since $|\{\alpha<\beta \mid h(\alpha) \leq \epsilon\}|<\mu$ for all $\beta \in b$, and $|b|<\mu$, let us pick $a \in \mathcal{A}^{\prime}$ such that $\min (h[a \times b])>\epsilon$.

Pick $\alpha, \beta \in \Gamma$ such that $a=a_{\alpha} \backslash z$ and $b=a_{\beta} \backslash z$. By Clause (d) above, we have $\alpha<\beta$ and $a_{\alpha} \cap a_{\beta}=z$. Then, $d\left(a_{\alpha}(i), a_{\beta}(i)\right)=\psi^{-1}\left(n^{*}, \tau^{*}, \gamma^{*}\right)$ whenever $\operatorname{otp}(z) \leq i<\chi$.

Suppose that $\eta \in u_{\alpha}$ and $\rho \in v_{\beta}$ are given, and write $\sigma:=\eta \frown \rho$.

(1) Since $\alpha \in \operatorname{Im}(\eta)$ and $\beta \in \operatorname{Im}(\rho)$, we infer the existence of a pair $\left(i^{\prime}, j^{\prime}\right)$ such that $i^{\prime}$ is the least to satisfy $\sigma\left(i^{\prime}\right)=\alpha$, and $j^{\prime}$ is the least to satisfy $\sigma\left(j^{\prime}\right)>\sup \left(a_{\alpha}\right)$. Then $\left(i^{\prime}, j^{\prime}\right) \in \mathcal{D}_{\sigma} \cap\left(\left(a_{\alpha} \backslash z\right) \times\left(a_{\beta} \backslash z\right)\right)$.

(2) By $\left(i^{\prime}, j^{\prime}\right) \in \mathcal{D}_{\sigma} \cap\left(\left(a_{\alpha} \backslash z\right) \times\left(a_{\beta} \backslash z\right)\right)$, we have

$$
\mathfrak{m}_{\sigma} \geq h\left(\sigma\left(i^{\prime}\right), \sigma\left(j^{\prime}\right)\right) \geq \min \left(h\left[\left(a_{\alpha} \backslash z\right) \times\left(a_{\beta} \backslash z\right)\right]\right)=\min (h[a \times b])>\epsilon .
$$

(3) Note that since $\alpha, \beta$ are distinct elements of $\operatorname{Im}(\sigma)$, we get that $\Delta_{\sigma} \geq$ $\Delta(\alpha, \beta)$. By Clause (a) above, $\alpha=a_{\alpha}\left(\chi^{\prime}\right)$ and $\beta=a_{\beta}\left(\chi^{\prime}\right)$, and so, by Clause (c) above, $\Delta(\alpha, \beta)>\epsilon$. In particular, $\Delta_{\sigma}>\epsilon$. So, by Clause (b) above, we should restrict our attention to the set

$$
\mathcal{I}:=\left\{(i, j) \in \chi \times \chi \mid \Delta\left(a_{\alpha}(i), a_{\beta}(j)\right)=\Delta_{\sigma}\right\}
$$

Let $(i, j)$ denote an arbitrary pair from $\mathcal{I}$. By $\epsilon<\Delta_{\sigma}<\infty$, we have $\epsilon<\Delta\left(a_{\alpha}(i), a_{\beta}(j)\right)<\infty$. By Clause (c) above, $f_{a_{\alpha}(j)} \uparrow(\epsilon+1)=$ $t(j)=f_{a_{\beta}(j)} \uparrow(\epsilon+1)$, and so if $i \neq j$, then $\Delta\left(a_{\alpha}(i), a_{\alpha}(j)\right)>\epsilon$, contradicting Clause (b) above. It follows that if $(i, j) \in \mathcal{I}$, then $i=j$. Let $(i, i) \in \mathcal{I}$ be arbitrary. By $\Delta\left(a_{\alpha}(i), a_{\beta}(i)\right)=\Delta_{\sigma}<\infty$, we get from Clause (d) above that $i \geq \operatorname{otp}(z)$, and then the choice of $\alpha, \beta$, entails that $d\left(a_{\alpha}(i), a_{\beta}(i)\right)=\psi^{-1}\left(n^{*}, \tau^{*}, \gamma^{*}\right)$. Altogether, $d_{0}(\sigma)=n^{*}, d_{1}(\sigma)=$ $\tau^{*}$ and $d_{2}(\sigma)=\gamma^{*}$.

Let $\alpha, \beta$ be given by the preceding claim. Suppose that we are given $\eta \in u_{\alpha}$ and $\rho \in v_{\beta}$, and let us show that $c_{0}(\eta \frown \rho)=\eta, c_{1}(\eta \frown \rho)=\alpha$, and $c_{2}(\eta \frown \rho)=\beta$. Indeed, the fact that $c_{3}(\eta \frown \rho)$ equals $\gamma^{*}$ already follows from the preceding claim. Write $\sigma:=\eta \frown \rho$. 
Claim 2.6.2. If $(i, j) \in \mathcal{P}_{\sigma}$, then:

(1) $\sigma(i) \in a_{\alpha} \backslash z$, and $\sigma(j) \in a_{\beta} \backslash z$;

(2) $j \geq \ell(\eta)$ and $i<\ell(\eta)$.

(3) $\left(i, \ell(\eta)+n^{*}\right) \in \mathcal{P}_{\sigma}$.

Proof. (1) By $\sigma=\eta \frown \rho$, we have $\{\sigma(i), \sigma(j)\} \subseteq \operatorname{Im}(\eta) \cup \operatorname{Im}(\rho) \subseteq a_{\alpha} \cup a_{\beta}$. If $\{\sigma(i), \sigma(j)\} \subseteq a_{\alpha}$, then $h(\sigma(i), \sigma(j)) \leq \sup \left(h^{\prime \prime}\left[a_{\alpha}\right]^{2}\right) \leq \epsilon$. Likewise, if $\{\sigma(i), \sigma(j)\} \subseteq a_{\beta}$, then $h(\sigma(i), \sigma(j)) \leq \sup \left(h "\left[a_{\beta}\right]^{2}\right) \leq \epsilon$. However, $h(\sigma(i), \sigma(j))=\mathfrak{m}_{\sigma}>\epsilon$. So, by $i<j$, we get that $\sigma(i) \in a_{\alpha}$ and $\sigma(j) \in$ $a_{\beta}$. Moreover, if $\sigma(j) \in z$, then $\{\sigma(i), \sigma(j)\} \subseteq a_{\alpha} \cup z=a_{\alpha}$, contradicting the fact that $\sup \left(h^{\prime \prime}\left[a_{\alpha}\right]^{2}\right)<\mathfrak{m}_{\sigma}$. So, $\sigma(j) \in a_{\beta} \backslash z$. Likewise, $\sigma(i) \in$ $a_{\alpha} \backslash z$.

(2) By $\sigma(j) \in a_{\beta} \backslash z=a_{\beta} \backslash a_{\alpha} \subseteq a_{\beta} \backslash \operatorname{Im}(\eta)$, we infer that $j \geq \ell(\eta)$. Likewise, $\sigma(i) \in a_{\alpha} \backslash z \subseteq a_{\alpha} \backslash \operatorname{Im}(\rho)$ and $i<\ell(\eta)$.

(3) Write $j^{*}:=\ell(\eta)+n^{*}$. Then, $i<\ell(\eta) \leq j^{*}$. By Clauses (e) and (d) above, we have $\sigma\left[\ell(\eta)+n^{*}\right] \subseteq a_{\alpha} \cup z \subseteq \sigma\left(j^{*}\right)$. Consequently, $\left(i, j^{*}\right) \in$ $\mathcal{D}_{\alpha}$.

Towards a contradiction, suppose that $h(\sigma(i), \sigma(j))>h\left(\sigma(i), \sigma\left(j^{*}\right)\right)$. In particular, $\sigma(j) \neq \sigma\left(j^{*}\right)$.

- If $\sigma(j)<\sigma\left(j^{*}\right)$, then by $(i, j) \in \mathcal{D}_{\sigma}$, it cannot be that $j^{*}<j$. Then, by the preceding item, we have $\ell(\eta) \leq j<j^{*}$. Consequently, there exists $n<n^{*}$ such that $j=\ell(\eta)+n$, and $\sigma(j)=\rho_{\beta}(n)$. By $\sigma(j) \in a_{\beta} \backslash z$ and Clause (d) above, $\sigma(j)>\sup (z)$. So $n$ contradicts the minimality of $n^{*}$.

- If $\sigma\left(j^{*}\right)<\sigma(j)$, then by $\left(\sigma(i), \sigma\left(j^{*}\right)\right) \in a_{\alpha} \times\left(a_{\beta} \backslash z\right)$ and Clause (d) above, we have $\sigma(i)<\sigma\left(j^{*}\right)<\sigma(j)$. By transitivity of $h$, then,

$$
h(\sigma(i), \sigma(j)) \leq \max \left\{h\left(\sigma(i), \sigma\left(j^{*}\right)\right), h\left(\sigma\left(j^{*}\right), \sigma(j)\right)\right\} .
$$

As we assume that $h(\sigma(i), \sigma(j))>h\left(\sigma(i), \sigma\left(j^{*}\right)\right)$, we must conclude that $h(\sigma(i), \sigma(j)) \leq h\left(\sigma\left(j^{*}\right), \sigma(j)\right) \leq \sup \left(h^{\prime \prime}\left[a_{\beta}\right]^{2}\right) \leq \epsilon<\mathfrak{m}_{\sigma}$, contradicting the fact that $(i, j) \in \mathcal{P}_{\sigma}$.

By Claim 2.6.2, Clauses (1) and (2), $\sigma\left(j_{\sigma}\right)>\sup (z)$ and $j_{\sigma} \geq \ell(\eta)$. Then, by minimality of $n^{*}$ and Claim 2.6.2(3), we have $j_{\sigma}=\ell(\eta)+n^{*}$. So, by 
Claim 2.6.1(3):

$$
c_{0}(\sigma)=\sigma \uparrow\left(j_{\sigma}-d_{0}(\sigma)\right)=(\eta \frown \rho) \uparrow\left(\left(\ell(\eta)+n^{*}\right)-n^{*}\right)=\eta .
$$

Finally, $c_{1}(\sigma)=\sigma\left(g_{d_{1}(\sigma)}\left(c_{0}(\sigma)\right)=(\eta \frown \rho)\left(g_{\tau^{*}}(\eta)\right)=\alpha\right.$, by Claim 2.6.1(3) and Clause (f) above. Likewise, $c_{2}(\sigma)=\rho\left(\left(g_{d_{1}(\sigma)}(\rho)\right)=\beta\right.$.

Note that the above $c$ is in particular a witness to $\mathrm{P} \ell_{6}\left(\mu^{+}, \mu^{+}, \mu\right)$, where:

Definition 2.7. $\mathrm{P} \ell_{6}(\kappa, \theta, \chi)$ asserts the existence of a coloring $c:{ }^{<\omega} \kappa \rightarrow$ $\omega \times \kappa \times \kappa \times \theta$, satisfying the following. For every club $E$ in $\kappa$, every regressive map $\varphi: E \rightarrow \theta$, and every sequence $\left\langle\left(u_{\alpha}, v_{\alpha}, \rho_{\alpha}\right) \mid \alpha \in E\right\rangle$, that satisfies

(1) $u_{\alpha}$ and $v_{\alpha}$ are nonempty elements of $\left[{ }^{<\omega} \kappa\right]^{<\chi}$;

(2) $\alpha \in \operatorname{Im}(\eta)$ for all $\eta \in u_{\alpha}$;

(3) $\rho_{\alpha} \frown\langle\alpha\rangle \sqsubseteq \rho$ for all $\rho \in v_{\alpha}$,

there exist $\alpha<\beta$ in $E$ with $\varphi(\alpha)=\varphi(\beta)$ such that $c(\eta \frown \rho)=(\ell(\eta), \alpha, \beta$, $\varphi(\beta))$ for all $\eta \in u_{\alpha}$ and $\rho \in v_{\beta}$.

Why is this useful? As the construction of $c$ anyway assumed $\mu^{<\mu}=\mu$, we have in this case [1] a sequence $\left\langle g_{i}:\left\langle\omega \mu^{+} \rightarrow \mu \mid i<\mu\right\rangle\right.$ such that for every $x \in\left[{ }^{<\omega} \mu^{+}\right]^{<\mu}$ and $g: x \rightarrow \mu$, one can find $i<\mu$ with $g \subseteq g_{i}$. So, we can encode into $\varphi(\beta)$ the value $i$ for which $g_{i} \uparrow u_{\beta} \cup v_{\beta}$ is as desired. As $\varphi(\alpha)=\varphi(\beta)$, this would, in addition, entail that $g_{i}\left\lceil u_{\alpha} \cup v_{\alpha}\right.$ is as desired. On top of that, we can use $\beta$ to stretch the function $g_{i}$ into a function $g_{i}^{\beta}$ with range $\omega \times \beta$. So, yielding $g_{i}^{\beta}(\eta)=\left(\ell(\eta), \gamma^{*}\right)$ is simply a special case.

Now, a second look at the arguments of Theorem 2.3 makes it clear that $\mathrm{P} \ell_{6}\left(\mu^{+}, \omega, \mu\right)$ holds for every regular accessible $\mu$. Altogether:

Corollary 2.8. $\mathrm{P} \ell_{6}\left(\mu^{+}, \omega, \mu\right)$ holds for every regular cardinal $\mu$.

Remark. For an application of $\mathrm{P} \ell_{6}\left(\mu^{+}, \omega, \mu\right)$, see [9].

Note that the arguments of Theorem 2.3 can be used to obtain additional results. For instance, $\mathrm{P} \ell_{6}(\lambda, \theta, \operatorname{cf}(\lambda))$ holds for every prevalent singular cardinal $\lambda$, and $\theta<\lambda .{ }^{5}$ Likewise, $\mathrm{P} \ell_{6}(\kappa, \theta, \chi)$ holds for every strongly inaccessible cardinal $\kappa$, and every $\theta, \chi<\kappa$.

\footnotetext{
${ }^{5}$ The notion of a prevalent cardinal was coined in [7].
} 


\section{The coloring principle $\operatorname{Pr}_{1}$}

In this section, $\mathrm{Pr}_{1}$ colorings are derived from $\mathrm{P} \ell_{6}$. Following the advice of one of the referees, we say a few words about the involved ingredients.

Suppose that $\theta$ is a regular uncountable cardinal. For every pair of ordinals $\alpha<\beta$ below $\theta$, one attaches a finite subset $T(\alpha, \beta)$ of the interval $[\alpha, \beta]$. Specifically, $T(\alpha, \beta)$ is the upper trace of the walk from $\beta$ down to $\alpha$. Given a nonreflecting subset $\Gamma$ of $\theta$, one can define the upper trace in such a way that for every unbounded subset $A$ of $\theta$, the set $\Gamma \backslash \bigcup\left\{T(\alpha, \beta) \mid(\alpha, \beta) \in[A]^{2}\right\}$ will be nonstationary. In particular, if $\Gamma$ is stationary, and $\psi: \Gamma \rightarrow \theta$ is a partition of $\Gamma$ into $\theta$ many stationary set, then for every unbounded subset $A$ of $\theta$, and every desired color $\gamma^{*}<\theta$, there exists $\alpha<\beta$ in $A$ and $\epsilon \in T(\alpha, \beta)$ such that $\psi(\epsilon)=\gamma^{*}$.

This suggests that a strong coloring $c:[\theta]^{2} \rightarrow \theta$ may be obtained by letting $c(\alpha, \beta)=\psi(\bar{\epsilon}(\alpha, \beta))$ where $\bar{\epsilon}(\alpha, \beta)$ is a cleverly chosen element of $T(\alpha, \beta)$. Since $T(\alpha, \beta)$ is a finite set, any choice function $\bar{\epsilon}$ in $\prod_{\alpha<\beta<\theta} T(\alpha, \beta)$ may be identified with a function $o$ in $\prod_{\alpha<\beta<\theta}|T(\alpha, \beta)|$. So, in summary, our challenge in this setup, is to come up with a useful oscillation function of the form $o:[\theta]^{2} \rightarrow \omega$. In [20], where the method of walks on ordinals was introduced, Todorčević obtained strong colorings by appealing to the socalled oscillation of the upper trace. In a recent paper [10], Todorčević and the author obtained strong colorings by appealing to the so-called oscillation of the lower trace. In many of Shelah's paper that were mentioned in the introduction, strong colorings are obtained by exhibiting very complicated oscillation functions. In this paper, an oscillation function will be derived from $\mathrm{P} \ell_{6}$ of the previous section, by feeding it with a projected version of the upper trace.

Theorem 3.1. Suppose that $\mathrm{P} \ell_{6}(\kappa, \chi)$ holds with $\chi \leq \kappa$. Suppose that $\theta>\kappa$ is a regular cardinal, and there exists a nonreflecting stationary subset of $E_{\geq \chi}^{\theta}$. Then $\operatorname{Pr}_{1}(\theta, \theta, \theta, \chi)$ holds.

Proof. Compared to previous works by Shelah [17, Section 4], [18, Section 1] and Todorčević [21, Section 8], the new feature here is the ability to take advantage of a $(\kappa, \chi)$-oscillation oracle, even when the nonreflection concentrates at points of cofinality $<\kappa$.

The coloring. Let $\Gamma \subseteq E_{\geq \chi}^{\theta}$ be stationary and nonreflecting. Let $\left\langle C_{\alpha}\right| \alpha<$ $\theta\rangle$ be a sequence such that $C_{\alpha+1}=\{\alpha\}$ for every $\alpha<\theta$, and such that for every limit $\alpha<\theta, C_{\alpha}$ is a club in $\alpha$ with $\Gamma \cap \operatorname{acc}\left(C_{\alpha}\right)=\emptyset$. Fix a surjection 
$h: \theta \rightarrow \kappa$ such that $H_{i}:=\{\alpha \in \Gamma \mid h(\alpha)=i\}$ is stationary for all $i<\kappa$. For all $\alpha<\beta<\theta$, define:

- $\operatorname{Tr}(\alpha, \beta) \in{ }^{\omega} \theta$, by recursively letting for all $n<\omega$ :

$$
\operatorname{Tr}(\alpha, \beta)(n):= \begin{cases}\beta, & n=0 \\ \min \left(C_{\operatorname{Tr}(\alpha, \beta)(n-1) \backslash \alpha),}\right. & n>0 \& \operatorname{Tr}(\alpha, \beta)(n-1)>\alpha \\ \alpha, & \text { otherwise }\end{cases}
$$

- $\rho^{2}(\alpha, \beta):=\min \{n<\omega \mid \operatorname{Tr}(\alpha, \beta)(n)=\alpha\} ;^{6}$

- $\operatorname{tr}_{\mathrm{h}}(\alpha, \beta):=\left\langle h(\operatorname{Tr}(\alpha, \beta)(i)) \mid i<\rho^{2}(\alpha, \beta)\right\rangle$;

- $\lambda(\alpha, \beta):=\max \left\{\sup \left(C_{\operatorname{Tr}(\alpha, \beta)(i)} \cap \alpha\right) \mid i<\rho^{2}(\alpha, \beta)\right\}$.

We remind the reader of two fundamental facts:

Claim 3.1.1. If $\delta \in \Gamma$ and $\delta<\beta<\theta$, then $\lambda(\delta, \beta)<\delta$.

Proof. If $\delta \geq \lambda(\delta, \beta)$, then by definition, there exists $i<\rho^{2}(\delta, \beta)$ such that $\sup \left(C_{\operatorname{Tr}(\delta, \beta)(i)} \cap \delta\right)=\delta$. In particular, there exists an ordinal $\alpha$ with $\delta<\alpha<$ $\beta$ such that $\sup \left(C_{\alpha} \cap \delta\right)=\delta$, contradicting the fact that $\operatorname{acc}\left(C_{\alpha}\right) \cap \Gamma=\emptyset$ for every $\alpha<\theta$.

Claim 3.1.2. If $\lambda(\beta, \gamma)<\alpha<\beta<\gamma<\theta$, then

$$
\operatorname{tr}_{\mathrm{h}}(\alpha, \gamma)=\operatorname{tr}_{\mathrm{h}}(\beta, \gamma) \frown \operatorname{tr}_{\mathrm{h}}(\alpha, \beta)
$$

Proof. Clearly, $\operatorname{Tr}(\alpha, \gamma)(0)=\gamma=\operatorname{Tr}(\beta, \gamma)(0)$. Next, if $i<\rho^{2}(\beta, \gamma)$ and $\operatorname{Tr}(\alpha$, $\gamma)(i)=\operatorname{Tr}(\beta, \gamma)(i)$, then by $\beta>\alpha>\lambda(\beta, \gamma) \geq \sup \left(C_{\operatorname{Tr}(\beta, \gamma)(i)} \cap \beta\right)$, we get that

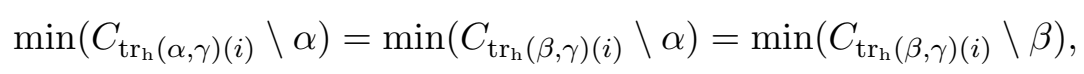

and hence $\operatorname{Tr}(\alpha, \gamma)(i+1)=\operatorname{Tr}(\beta, \gamma)(i+1)$. So $\operatorname{Tr}(\beta, \gamma) \uparrow \rho^{2}(\beta, \gamma) \sqsubseteq \operatorname{Tr}(\alpha$, $\gamma)$, and $\operatorname{Tr}(\alpha, \gamma)\left(\rho^{2}(\beta, \gamma)\right)=\beta$, let alone $\operatorname{tr}_{\mathrm{h}}(\alpha, \gamma)=\operatorname{tr}_{\mathrm{h}}(\beta, \gamma) \frown \operatorname{tr}_{\mathrm{h}}(\alpha, \beta)$.

\footnotetext{
${ }^{6}$ The standard notation here is $\rho_{2}(\alpha, \beta)$. This time, we decided to use the notation $\rho^{2}$ to avoid confusion with the $\rho_{i}$ 's that are given by the definition of $\mathrm{P} \ell_{6}$.
} 
Let $d$ witness $\mathrm{P} \ell_{6}(\kappa, \chi)$. Fix a surjection $\psi: \theta \rightarrow \theta$ such that $\Gamma_{\gamma}:=\{\delta \in$ $\Gamma \mid \psi(\delta)=\gamma\}$ is stationary for every $\gamma<\theta$. Define $c:[\theta]^{2} \rightarrow \theta$ by stipulating

$$
c(\alpha, \beta):=\psi\left(\operatorname{Tr}(\alpha, \beta)\left(d\left(\operatorname{tr}_{\mathrm{h}}(\alpha, \beta)\right)\right)\right) .
$$

Let us describe the definition of $c$ in simple words: we first walk from $\beta$ down to $\alpha$, producing some sequence $\sigma$ in ${ }^{<\omega} \theta$. We then project it down using $h$ to a sequence $\bar{\sigma}$ in ${ }^{<\omega} \kappa$. We feed $\bar{\sigma}$ to $d$, and get some natural number $n$. By definition of $\mathrm{P} \ell_{6}$, we should think of $n$ as a number $<\ell(\bar{\sigma})$. As $n<\ell(\bar{\sigma})=\rho^{2}(\alpha, \beta)$, it then makes sense to look at the $n_{t h}$-step of the walk from $\beta$ down to $\alpha$ and ask about the color it was assigned by $\psi$. Indeed, this is what $c$ does.

Verification. Suppose that $\mathcal{A} \subseteq[\theta]^{<\chi}$ of size $\theta$, consists of pairwise disjoint sets, and $\gamma^{*}<\theta$ is a prescribed color. Let $\left\langle x_{\delta} \mid \delta<\theta\right\rangle$ be an injective enumeration of some subfamily of $\mathcal{A}$, such that $\delta<\min \left(x_{\delta}\right)$ for all $\delta<\theta$. Define a function $g: \Gamma \rightarrow \theta$ by letting

$$
g(\delta):=\sup \left\{\lambda(\delta, \beta) \mid \beta \in x_{\delta}\right\} .
$$

By Claim 3.1.1 and $\left|x_{\delta}\right|<\operatorname{cf}(\delta)$ for all $\delta \in \Gamma, g$ is regressive, so for all $i<\kappa$, let us pick a stationary subset $\Sigma_{i} \subseteq H_{i}$ such that $g\left\lceil\Sigma_{i}\right.$ is constant.

Put $D:=\left\{\delta<\theta \mid \forall \alpha<\delta\left(\sup \left(x_{\alpha}\right)<\delta\right)\right\}$. Denote $\Delta_{j}:=H_{j} \cap D$, and fix $\epsilon \in \Gamma \cap \bigcap_{j<\kappa} \operatorname{acc}^{+}\left(\Delta_{j}\right)$ above $\zeta:=\sup \left(g\left[\bigcup_{i<\kappa} \Sigma_{i}\right]\right)$ such that $\psi(\epsilon)=\gamma^{*}$. For all $i<\kappa$, let

$$
\beta_{i}:=\min \left(\Sigma_{i} \backslash \epsilon+1\right) .
$$

- If $\operatorname{cf}(\epsilon) \geq \kappa$, let $\varphi: \kappa \rightarrow\{0\}$ be the constant function.

Then, for each $j<\kappa$, by $\epsilon \in \Gamma \cap \operatorname{acc}^{+}\left(\Delta_{j}\right)$ and Claim 3.1.1, pick $\delta_{j} \in \Delta_{j}$ such that

- $\epsilon>\delta_{j}>\max \left\{\sup \left\{\lambda(\epsilon, \beta) \mid \beta \in x_{\beta_{i}}, i<j\right\}, \zeta\right\}$, and put

- $\alpha_{j}:=\max \left\{\sup \left\{\lambda(\epsilon, \beta) \mid \beta \in x_{\beta_{i}}, i<j\right\}, \zeta, \lambda\left(\delta_{j}, \epsilon\right)\right\}+1$.

- If $\chi \leq \operatorname{cf}(\epsilon)<\kappa$, let $\left\langle\epsilon_{\iota} \mid \iota<\operatorname{cf}(\epsilon)\right\rangle$ be an increasing sequence of ordinals converging to $\epsilon$. Define $\varphi: \kappa \rightarrow \operatorname{cf}(\epsilon)$ by stipulating

$$
\varphi(i):=\min \left\{\iota<\operatorname{cf}(\epsilon) \mid \sup \left\{\lambda(\epsilon, \beta) \mid \beta \in x_{\beta_{i}}\right\}<\epsilon_{\iota}\right\} .
$$

Then, for each $j<\kappa$, by $\epsilon \in \operatorname{acc}^{+}\left(\Delta_{j}\right)$, pick $\delta_{j} \in \Delta_{j}$ such that

- $\epsilon>\delta_{j}>\max \left\{\epsilon_{\varphi(j)}, \zeta\right\}$, and put

- $\alpha_{j}:=\max \left\{\epsilon_{\varphi(j)}, \zeta, \lambda\left(\delta_{j}, \epsilon\right)\right\}+1$.

Write $a_{j}:=x_{\alpha_{j}}$ and $b_{i}:=x_{\beta_{i}}$. 
Claim 3.1.3. For every $i<j<\kappa$ and every $\alpha \in a_{j}, \beta \in b_{i}$, we have:

(a) $\operatorname{tr}_{\mathrm{h}}(\epsilon, \beta)=\operatorname{tr}_{\mathrm{h}}\left(\beta_{i}, \beta\right) \frown \operatorname{tr}_{\mathrm{h}}\left(\epsilon, \beta_{i}\right)$;

(b) $\operatorname{tr}_{\mathrm{h}}(\alpha, \epsilon)=\operatorname{tr}_{\mathrm{h}}\left(\delta_{j}, \epsilon\right) \frown \operatorname{tr}_{\mathrm{h}}\left(\alpha, \delta_{j}\right)$;

(c) $\operatorname{tr}_{\mathrm{h}}(\alpha, \beta)=\operatorname{tr}_{\mathrm{h}}(\epsilon, \beta) \frown \operatorname{tr}_{\mathrm{h}}(\alpha, \epsilon)$, provided that $\varphi(i)=\varphi(j)$.

Proof. All clauses follow from Claim 3.1.2 and the following particular considerations.

(a) By $\beta \in b_{i}=x_{\beta_{i}}$, we have $\beta>\beta_{i}>\epsilon>\zeta \geq g\left(\beta_{i}\right) \geq \lambda\left(\beta_{i}, \beta\right)$.

(b) By $\delta_{j} \in \Gamma$ and Claim 3.1.1, the definition of $\alpha_{j}$ makes it clear that $\delta_{j}>$ $\alpha_{j}>\lambda\left(\delta_{j}, \epsilon\right)$. By $\delta_{j} \in D$, we have $\delta_{j}>\sup \left(x_{\alpha_{j}}\right)$. So, by $\alpha \in a_{j}=x_{\alpha_{j}}$, we get $\epsilon>\delta_{j}>\alpha>\alpha_{j}>\lambda\left(\delta_{j}, \epsilon\right)$.

(c) If $\operatorname{cf}(\epsilon) \geq \kappa$, then we have $\alpha_{j}>\sup \left\{\lambda\left(\epsilon, \beta^{\prime}\right) \mid \beta^{\prime} \in x_{\beta_{i^{\prime}}}, i^{\prime}<j\right\}$. And so by $i<j, \beta \in b_{i}=x_{\beta_{i}}$ and $\alpha \in a_{j}=x_{\alpha_{j}}$, we have $\beta>\beta_{i}>\epsilon>\delta_{j}>$ $\alpha>\alpha_{j}>\lambda(\epsilon, \beta)$.

If $\operatorname{cf}(\epsilon)<\kappa$, then by $\varphi(i)=\varphi(j)$, we get $\alpha_{j}>\epsilon_{\varphi(j)}=\epsilon_{\varphi(i)}>$ $\sup \left\{\lambda\left(\epsilon, \beta^{\prime}\right) \mid \beta^{\prime} \in x_{\beta_{i}}\right\}$. And so by $\beta \in b_{i}=x_{\beta_{i}}$ and $\alpha \in a_{j}=x_{\alpha_{j}}$, we have $\beta>\beta_{i}>\epsilon>\delta_{j}>\alpha>\alpha_{j}>\lambda(\epsilon, \beta)$.

For every $i<\kappa$, set $u_{i}:=\left\{\operatorname{tr}_{\mathrm{h}}(\epsilon, \beta) \mid \beta \in b_{i}\right\}$ and $v_{i}:=\left\{\operatorname{tr}_{\mathrm{h}}(\alpha, \epsilon) \mid\right.$ $\left.\alpha \in a_{i}\right\}$.

Claim 3.1.4. For every $i<\kappa$ :

(1) $i \in \operatorname{Im}(\eta)$ for all $\eta \in u_{i}$;

(2) there exists $\rho_{i} \in{ }^{\langle\omega} \kappa$ such that $\rho_{i} \frown\langle i\rangle \sqsubseteq \rho$ for all $\rho \in v_{i}$.

Proof. (1) By Claim 3.1.3(a), and the fact that $\beta_{i} \in \Sigma_{i} \subseteq H_{i}$.

(2) By Claim 3.1.3(b) and $\delta_{i} \in \Delta_{i} \subseteq H_{i}$, we have $\operatorname{tr}_{\mathrm{h}}\left(\delta_{i}, \epsilon\right) \frown\langle i\rangle \sqsubseteq \rho$ for all $\rho \in v_{i}$.

By the choice of $d$, then, there exist $i<j<\kappa$ with $\varphi(i)=\varphi(j)$ such that $d(\eta \frown \rho)=\ell(\eta)$ for all $\eta \in u_{i}$ and $\rho \in v_{j}$. To see that $c\left[a_{j} \times b_{i}\right]=\left\{\gamma^{*}\right\}$, fix an arbitrary $\alpha \in a_{j}$ and $\beta \in b_{i}$. Write $\eta:=\operatorname{tr}_{\mathrm{h}}(\epsilon, \beta)$ and $\rho:=\operatorname{tr}_{\mathrm{h}}(\alpha, \epsilon)$. Then:

- $\operatorname{tr}_{\mathrm{h}}(\alpha, \beta)=\eta \frown \rho$, by Claim 3.1.3(c); 
- $d\left(\operatorname{tr}_{\mathrm{h}}(\alpha, \beta)\right)=\ell(\eta)=\ell\left(\operatorname{tr}_{\mathrm{h}}(\epsilon, \beta)\right)=\rho^{2}(\epsilon, \beta)$;

- $\operatorname{Tr}(\alpha, \beta)\left(d\left(\operatorname{tr}_{\mathrm{h}}(\alpha, \beta)\right)\right)=\operatorname{Tr}(\alpha, \beta)\left(\rho^{2}(\epsilon, \beta)\right)=\epsilon$, by Claim 3.1.3(c).

So, $c(\alpha, \beta)=\psi\left(\operatorname{Tr}(\alpha, \beta)\left(d\left(\operatorname{tr}_{\mathrm{h}}(\alpha, \beta)\right)\right)\right)=\psi(\epsilon)=\gamma^{*}$, as desired.

Corollary 3.2. If $\lambda=\operatorname{cf}(\lambda)>\kappa^{+}$, and $E_{\geq \kappa}^{\lambda}$ admits a nonreflecting stationary set, then $\operatorname{Pr}_{1}(\lambda, \lambda, \lambda, \chi)$ holds for every regular cardinal $\chi \leq \kappa$.

Proof. Suppose that $\chi \leq \kappa$ is regular. Then, by Theorem 2.2, $\mathrm{P} \ell_{6}\left(\chi^{+}, \chi\right)$ holds. As $\lambda>\chi^{+}$, and $E_{\geq \chi}^{\lambda}$ admits a nonreflecting stationary set, we get $\operatorname{Pr}_{1}(\lambda, \lambda, \lambda, \chi)$ as a consequence of Theorem 3.1.

\section{Acknowledgements}

I thank M. Kojman and D. Soukup for stimulating discussions on [18], and of course, the author of [18], S. Shelah, for serving as an endless source of inspiration. I also thank the referees for their thoughtful feedback.

The results of this paper were announced in a talk in the Set Theory workshop in Oberwolfach, January 2014. The proofs were subsequently presented in detail through two informal sessions; I thank J. Cummings, T. Eisworth, J.T. Moore, D. Raghavan, S. Todorčević and B. Veličković for their comments, objections and suggestions.

I am grateful to M. Gitik for detecting an error in one of the early versions of my proof.

Partially supported by ISF grant 1630/14.

\section{References}

[1] R. Engelking and M. Karłowicz, Some theorems of set theory and their topological consequences, Fund. Math. 57 (1965), 275-285.

[2] P. Erdős, A. Hajnal and R. Rado, Partition relations for cardinal numbers, Acta Math. Acad. Sci. Hungar. 16 (1965), 93-196.

[3] D.H. Fremlin, Consequences of Martin's axiom, in 'Cambridge Tracts in Mathematics', 84, Cambridge University Press, Cambridge, 1984, ISBN 0-521-25091-9.

[4] F. Galvin, Chain conditions and products, Fund. Math. 108(1) (1980), 33-48. 
[5] A. Hajnal and I. Juhász, A consequence of Martin's axiom, Nederl. Akad. Wetensch. Proc. Ser. A 74=Indag. Math. 33 (1971), 457-463.

[6] K. Kunen, Set theory, in 'Studies in Logic and the Foundations of Mathematics', 102, North-Holland Publishing Co., Amsterdam-New York, 1980, ISBN 0-444-85401-0. An introduction to independence proofs.

[7] A. Rinot, On topological spaces of singular density and minimal weight, Topol. Appl. 155(3) (2007), 135-140.

[8] - Transforming rectangles into squares, with applications to strong colorings, Adv. Math. 231(2) (2012), 1085-1099.

[9] - Chain conditions of products, and weakly compact cardinals, B. Symbolic Logic 20(3) (2014), 293-314.

[10] A. Rinot and S. Todorčević, Rectangular square-bracket operation for successor of regular cardinals, Fund. Math. 220(2) (2013), 119-128.

[11] S. Shelah, A graph which embeds all small graphs on any large set of vertices, Ann. Pure Appl. Logic 38 (1988), 171-183.

[12] - Successors of singulars, cofinalities of reduced products of cardinals and productivity of chain conditions, Israel J. Math. 62 (1988), 213-256.

[13] — Was Sierpiński right? I, Israel J. Math. 62 (1988), 355-380.

[14] - Strong negative partition above the continuum, J. Symbolic Logic 55 (1990), 21-31.

[15] - Strong negative partition relations below the continuum, Acta Math. Hungar. 58 (1991), 95-100.

[16] — Cardinal arithmetic, in 'Oxford Logic Guides' 29, The Clarendon Press, Oxford University Press, New York, 1994, ISBN 0-19-8537859, Oxford Science Publications.

[17] - There are Jonsson algebras in many inaccessible cardinals, in Cardinal Arithmetic, in 'Oxford Logic Guides', 29, Oxford University Press, 1994.

[18] - Colouring and non-productivity of $\aleph_{2}-c c$, Ann. Pure Appl. Logic 84 (1997), 153-174.

[19] - The colouring existence theorem revisited, 2013, arXiv:1311.1026. 
[20] S. Todorčević, Partitioning pairs of countable ordinals, Acta Math. 159(3-4) (1987), 261-294.

[21] - Walks on ordinals and their characteristics, 'Progress in Mathematics', 263, Birkhäuser Verlag, Basel, 2007, ISBN 978-3-7643-8528-6.

Department of Mathematics

BAR-ILAN UNIVERSITY

RAMAT-GAN 52900

ISRAEL

E-mail address: rinotas@math.biu.ac.il

URL : http://www.assafrinot.com

RECEIVEd January 19, 2014 